\title{
Exogenous isoleucine and phenylalanine interact with abscisic acid-mediated anthocyanin accumulation in grape
}

\author{
Tomoki Hattori', Yang Chen', Shinichi Enoki', \\ Daisuke Igarashi², Shunji Suzuki*
}

\author{
${ }^{1}$ The Institute of Enology and Viticulture, University of Yamanashi, Kofu, Yamanashi, Japan \\ ${ }^{2}$ Frontier Research Labs, Institute for Innovation, Ajinomoto Co., Inc., Kawasaki, Kanagawa, Japan
}

\begin{abstract}
Berry skin colour is a crucial determinant of red/black grape berry quality. We investigated the effects of combination treatments with amino acids and a low concentration of ABA on anthocyanin accumulation in grapes. Among the amino acids tested, isoleucine and phenylalanine resulted in high anthocyanin contents in grape cell cultures. The combination treatments with isoleucine or phenylalanine, and a low concentration of ABA enhanced anthocyanin accumulation in grape cells and detached grape berries. The combination treatment with isoleucine, but not with phenylalanine, and ABA upregulated MybA1 expression. Field-grown grapevines received combination treatments with isoleucine or phenylalanine, and ABA in two growing seasons. In the 2015 growing season, the combination treatments with isoleucine or phenylalanine, and a low concentration of ABA accelerated anthocyanin accumulation in grape berry skins of field-grown grapevines on days 10 and 31 post treatment. The effects on anthocyanin accumulation became negligible at harvest. The effect of the combination treatment with phenylalanine and a low concentration of ABA on anthocyanin accumulation was masked in the 2017 growing season due to the unexpected stimulation of anthocyanin accumulation by the low concentration of ABA, although the combination treatment accelerated anthocyanin accumulation on days 3 and 10 post treatment. Taken together, the results suggested that exogenous isoleucine and phenylalanine interacted with ABA-mediated anthocyanin accumulation in grape berry skins of fieldgrown grapevines when the activity of ABA used to treat the grapevines was inadequate.
\end{abstract}

Key words: abscisic acid, anthocyanin, grapevine, isoleucine, phenylalanine

\section{Abbreviations:}

ABA - abscisic acid, UFGT - UDP glucose flavonoid 3-O-glucosyltransferase

\section{INTRODUCTION}

The major objectives in viticulture are to increase the quality as well as health benefits of grape berry (Gąstoł, 2015). Grape berry skin colour, determined by the anthocyanin content, is a crucial determinant of red/black grape berry quality, and consequently, red wine quality. Anthocyanin is a key contributor to the colour of young red wine, while more stable pigments of anthocyanins with other polyphenols are responsible for the colour of aged red wine (Castellarin et al., 2006; Castellarin and Gaspero, 2007). Although there are differences in anthocyanin extractability from grapes to wines according to varieties (Romero-Cascales et al., 2005), a higher anthocyanin content in grape

\footnotetext{
*Corresponding author. 
berry skins generally results in a higher wine colour intensity. Anthocyanins are accumulated in grape berry skins during fruit ripening and their concentration in grape berry skin is clearly affected by growing conditions (Kennedy et al., 2006). For example, a high temperature inhibited anthocyanin accumulation in grape berry skin (Mori et al., 2005; Mori et al., 2007). Model simulation of climate change in viticulture regions predicted an average warming of $2^{\circ} \mathrm{C}$ in the next 50 years (Jones et al., 2005). Therefore, global warming will pose a grave problem for viticulturists in terms of grape berry skin coloration in the near future.

A number of practical techniques have been developed to maintain and improve grape berry skin colour. Viticultural practices, such as leaf removal (Matus et al., 2009), cluster thinning (Guidoni et al., 2002), and girdling (Brar et al., 2008), can change the anthocyanin content in grape berry skin. Application of chemicals might offer an alternative way to improve the anthocyanin content in grape berry skin. Foliar treatments with methyl jasmonate increased the anthocyanin content in grape berry skin (Portu et al., 2016). Exogenous abscisic acid (ABA), a phytohormone, rapidly improved grape berry skin colour (Peppi et al., 2008). The application of chemicals to grapevines also stimulates endogenous ABA biosynthesis in grape berry. Vanillylacetone upregulated gene expression leading to anthocyanin biosynthesis through the induction of endogenous ABA, resulting in a rapid anthocyanin accumulation in grape berry skin (Enoki et al., 2017). In general, ABA biosynthesis occurred in grape berry during ripening (Deluc et al., 2007). Endogenous ABA content increased drastically from véraison (Gagné et al., 2006) and accelerated grape berry ripening, including anthocyanin biosynthesis (Pilati et al., 2017). Thus, to maintain and improve grape berry skin colour, upregulation of ABA content in grape berry skins is definitely required.

However, synthetic ABA is expensive for practical field application in viticulture. In the present study, to reduce the application volume of ABA by using a combination of inexpensive amino acids and ABA, we tested whether the application of amino acids interacts with ABAmediated anthocyanin accumulation in grape cells. Amino acids play important roles in plant growth and secondary metabolite production (Calvo et al., 2014). The application of amino acids to grapevine is generally accomplished through the use of fertilizers to increase nitrogen-containing substances in grape berry (Smit et al., 2014). Foliar treatment with phenylalanine affected the phenolic content and aroma composition in grapevine (Garde-Cerdán et al., 2014; Portu et al., 2015). Based on these scientific findings, amino acids have been used in viticulture as biostimulants for physiological state or supplements for nitrogen source. We herein show that the combination treatment with isoleucine or phenylalanine, selected in the present study, and a low concentration of ABA synergistically enhanced anthocyanin accumulation in grape cell cultures and grape berry skins.

\section{MATERIALS AND METHODS}

\section{Chemicals}

Isoleucine, phenylalanine, and other amino acids were obtained from Ajinomoto Co., Inc. (Tokyo, Japan). Abscisic acid (ABA) was purchased from Tokyo Chemical Industry Co., Ltd. (Tokyo, Japan).

\section{Plant materials}

VR cells, which are cultured cells derived from anthers of the Vitis interspecific hybrid cultivar Bailey Alicante A, produce relatively and stably higher amounts of anthocyanins (2-3 $\mathrm{mg} \mathrm{g}^{-1}$ of fresh weight) (Yamakawa et al., 1983). The cultured cells were obtained from the RIKEN BioResource Center (Ibaraki, Japan) and were maintained on a modified Linsmaier and Skoog (LS) agar medium (pH 5.7) supplemented with $3 \%$ sucrose, $0.2 \mathrm{mg} \mathrm{L}^{-1}$ kinetin, and $0.05 \mathrm{mg} \mathrm{mL}^{-1}$ 2,4-dichlorophenoxyacetic acid.

\section{Amino acid treatment of grapevine cultured cells}

VR cells were incubated on the modified LS agar medium and grown at $25^{\circ} \mathrm{C}$ in the dark for one month. To determine the effect of amino acids on anthocyanin biosynthesis in grape cell cultures, some VR cells were inoculated on the medium supplemented with $0.2,1$ or $5 \mathrm{mM}$ of each amino acid listed in Figure 1. VR cells were also inoculated on the medium supplemented with/without $0.1 \mathrm{mM}$ ABA as controls. After 5 days' incubation at $25^{\circ} \mathrm{C}$ under low light of $50 \mu \mathrm{mol} \mathrm{m} \mathrm{m}^{-2} \mathrm{~s}^{-1}$, the cells were frozen immediately in liquid nitrogen and subjected to anthocyanin measurements. The anthocyanin contents in the VR cells treated with each amino acid were expressed as relative values (percentage) in relation to the anthocyanin content in the VR cells treated with $0.2 \mathrm{mM}$ ABA.

\section{Combination treatment of grapevine cultured cells with isoleucine or phenylalanine, and $A B A$}

VR cells were inoculated on the medium supplemented with $10 \mathrm{mM}$ isoleucine or phenyl- 
alanine with $0.1 \mathrm{mM}$ ABA. VR cells were also inoculated on the medium supplemented with 0.1 $\mathrm{mM}$ ABA or used without supplementation as controls. After 5 days' incubation at $25^{\circ} \mathrm{C}$ under low light of $50 \mu \mathrm{mol} \mathrm{m}{ }^{-2} \mathrm{~s}^{-1}$, the cells were frozen immediately in liquid nitrogen and stored at $-80^{\circ} \mathrm{C}$ for further analyses.

\section{Combination treatment of detached grape berries with isoleucine or phenylalanine, and $A B A$}

Grape berries of Vitis vinifera cv. Cabernet Sauvignon cultivated in the experimental vineyard of The Institute of Enology and Viticulture, University of Yamanashi, Japan, were collected at véraison (Aug 10, 2017). The detached berries were washed with tap water and sterilized with $1 \% \mathrm{HClO}$ in $70 \%$ ethanol followed by air-drying. Ten $\mathrm{mM}$ isoleucine or phenylalanine with/without $1 \mathrm{mM}$ ABA was dissolved in $0.3 \mathrm{M}$ sucrose. The berries were placed on filter papers (Advantec Toyo No. 2, $90 \mathrm{~mm}$ diameter, Tokyo, Japan) soaked with the solution in 90 -mm-diameter Petri dishes. After treatment at $25^{\circ} \mathrm{C}$ for 8 days under low light of $50 \mu \mathrm{mol} \mathrm{m} \mathrm{m}^{-2} \mathrm{~s}^{-1}$, the berries were subjected to anthocyanin measurements.

\section{Combination treatment of field-grown grapevines with isoleucine or phenylalanine, and $A B A$}

To avoid a misunderstanding of field experimental data obtained by the same treatment on the same grapevine in the same vineyard every year, we performed field experiments every other year. Six field-grown grapevines were prepared for combination treatment with isoleucine or phenylalanine, and ABA in 2015 and 2017. $V$. vinifera cv. Cabernet Sauvignon vines, which were approximately 30 years old and trained to the Guyot-style system, cultivated in the experimental vineyard of the University of Yamanashi, Japan, were used. In this study, we treated the grapevines with isoleucine only, phenylalanine only, ABA only, isoleucine plus ABA, or phenylalanine plus ABA. Non-treated grapevines served as control. The treatments were carried out at véraison (Jul 31, 2015 and Aug 10, 2017). Briefly, all grape bunches on a grapevine were dipped into $10 \mathrm{mM}$ isoleucine or phenylalanine with/without $380 \mu \mathrm{M}$ ABA. The applications of ABA $(380 \mu \mathrm{M})$ only were used as control treatments. Before treatment, 0.1\% Approach BI (Kao, Wakayama, Japan) as a surfactant was added to all the treatment agents. Three grape bunches were collected from each treatment on day $0,3,10,31$, and 66 (in the 2015 growing season), or 64 (in the 2017 growing season) post application. Thirty fresh berries were randomly sampled from each bunch and subjected to anthocyanin measurements.

\section{Total RNA isolation}

VR cells were frozen in liquid nitrogen and homogenized using an SK mill (SK-200, Tokken, Kashiwa, Japan). Total RNA isolation from the pulverized cells was performed with a Fruit-mate for RNA Purification (Takara, Otsu, Japan) and a NucleoSpin RNA Plant (Takara) according to the manufacturer's instructions (Mikami et al., 2017).

\section{Real-time RT-PCR analysis}

cDNA synthesis and real-time RT-PCR were performed as described previously (Arita et al., 2017). Briefly, cDNA was synthesized from total RNA using a PrimeScript RT Reagent Kit with gDNA Eraser (Takara) according to the manufacturer's instructions. Real-time RT-PCR was performed with SYBR Premix Ex Taq II (Takara). PCR amplification was performed for 40 cycles at $95^{\circ} \mathrm{C}$ for $5 \mathrm{~s}$ and at $60^{\circ} \mathrm{C}$ for $1 \mathrm{~min}$. after initial denaturation at $95^{\circ} \mathrm{C}$ for $30 \mathrm{~s}$. The nucleotide sequences of the primers used for real-time RT-PCR were as follows: MybA1 primers (5'-GAAGTCCTCTTCCAGCCATCTC-3' and 5'-TTGACCCACCACTAAGCACAAT-3' from $V$. vinifera VvMybA1, GenBank accession no. XM_002282480), UDP glucose flavonoid 3-O-glucosyl transferase (UFGT) primers (5' -TCACTCTCCACCCATGTTTACACT-3' and 5'-AATTGAGCAGCTCGTCTTCACG-3', from $V$. vinifera UFGT, GenBank accession no. XM_0022769994), and actin primers (5' -ATTÄGGCTTCACAATTTGTTGGGG-3' and 5'-TATCTCTCCCTTCTTCCTGGAACT-3', from $V$. vinifera actin 1, GenBank accession no. XM_010666609). Actin was used for data normalization. The dissociation curves were evaluated to verify the specificity of the amplification reaction. The expression levels of each gene were determined as the number of amplification cycles needed to reach a fixed threshold by the standard curve method with Thermal Cycler Dice Real Time System Single Software ver. 3.00 (Takara). Data were expressed as relative values in relation to actin and presented as means \pm standard errors.

\section{Anthocyanin measurement}

Skins were peeled off from grape berries using tweezers. Anthocyanin extraction from VR cells and grape berry skins and its measurement were performed as described previously (Yokotsuka 
et al., 1999), with modification. Briefly, the samples were pulverized in liquid nitrogen. One gram of the pulverized sample was macerated in $20 \mathrm{~mL}$ of $0.1 \mathrm{~N} \mathrm{HCl}$ for $4 \mathrm{~h}$ at room temperature in the dark and filtered through a $0.45 \mu \mathrm{m}$ cellulose acetate filter (Advantec Toyo, Tokyo, Japan). Absorbance (OD520) of the filtrate was measured using a spectrometer (UV-1800, Shimadzu, Kyoto, Japan). Total anthocyanin content was calculated by a previously published formula (Bakker et al., 1986) and converted into malvidin-3-glucoside equivalents as $\mu \mathrm{g}$ per gram of fresh weight of cell mass for VR cells, and mg per gram of fresh skin weight for grape berry skins.

\section{Statistical analysis}

Data are presented as means \pm standard errors calculated from the indicated biological replicates. Statistical analysis was carried out by Tukey's multiple comparison test using Excel statistics software 2012 (Social Survey Research Information, Tokyo, Japan).

\section{RESULTS}

\section{Isoleucine and phenylalanine partially enhance anthocyanin accumulation in VR cells}

Tyrosine was not determined due to its low solubility. VR cells were treated with 19 amino acids for 5 days. Anthocyanin contents were calculated as relative values in relation to the anthocyanin content in the VR cells treated with ABA. Although the amount of anthocyanin accumulation induced

\begin{tabular}{|c|c|c|c|}
\hline & $0.2 \mathrm{mM}$ & $1 \mathrm{mM}$ & $5 \mathrm{mM}$ \\
\hline Pro & 7 & 10 & 27 \\
\hline Ile & 11 & 26 & 76 \\
\hline Asp & 2 & 4 & 11 \\
\hline Glu & 11 & 14 & 4 \\
\hline Ser & 12 & 23 & 38 \\
\hline Asn & 11 & 9 & 38 \\
\hline Gln & 6 & 19 & 18 \\
\hline Gly & 19 & 15 & 23 \\
\hline His & 16 & 17 & 29 \\
\hline Arg & 10 & 5 & 9 \\
\hline ntrol & 12 & & \\
\hline ABA & 100 & & \\
\hline
\end{tabular}

by isoleucine or phenylalanine was less than that induced by $0.2 \mathrm{mM}$ ABA, $1 \mathrm{mM}$ and $5 \mathrm{mM}$ of isoleucine, phenylalanine, methionine, serine, leucine, and asparagine, among the amino acids tested, led to high anthocyanin contents in VR cells (Fig. 1). In the present study, we selected isoleucine and phenylalanine as the most significant amino acids to enhance anthocyanin accumulation.

\section{Isoleucine and phenylalanine interact with $A B A$ - mediated anthocyanin accumulation in VR cells}

To determine whether exogenous isoleucine or phenylalanine interacts with ABA-mediated anthocyanin accumulation, VR cells were subjected to combination treatments with isoleucine or phenylalanine, and a low concentration $(0.1 \mathrm{mM})$ of ABA (Fig. 2A). ABA at $0.1 \mathrm{mM}$ did not induce anthocyanin accumulation in VR cells (Fig. 2B). Isoleucine or phenylalanine alone also did not induce anthocyanin accumulation in VR cells. In contrast, VR cells that had received the combination treatment with isoleucine or phenylalanine, and ABA had significantly higher anthocyanin contents than the control and ABA-treated VR cells. Simultaneous treatment with isoleucine and phenylalanine had no effect on anthocyanin accumulation in VR cells.

Taken together, the results suggested that exogenous isoleucine and phenylalanine, used separately, interacted with a low concentration of ABA-mediated anthocyanin accumulation in VR cells.

\begin{tabular}{|c|c|c|c|}
\hline \multicolumn{2}{|c|}{$0.2 \mathrm{mM}$} & $1 \mathrm{mM}$ & $5 \mathrm{mM}$ \\
\hline Val & 12 & 7 & 6 \\
\hline et & 5 & 8 & 42 \\
\hline & 9 & 8 & 7 \\
\hline & 9 & 10 & 4 \\
\hline & 18 & 30 & 40 \\
\hline & 3 & 9 & 6 \\
\hline & 11 & 12 & 30 \\
\hline & 10 & 15 & 17 \\
\hline & 11 & 13 & 12 \\
\hline & n.d. & n.d. & n.d \\
\hline
\end{tabular}

Figure 1. Effect of amino acids on anthocyanin accumulation in grape cell cultures. VR cells were treated with $0.2,1$ or $5 \mathrm{mM}$ of each amino acid. Bars and numbers indicate relative values (percentage) of anthocyanin contents in relation to the anthocyanin content in VR cells treated with $0.2 \mathrm{mM} \mathrm{ABA}$. Control - no treatment, n.d. - not determined 
(A)

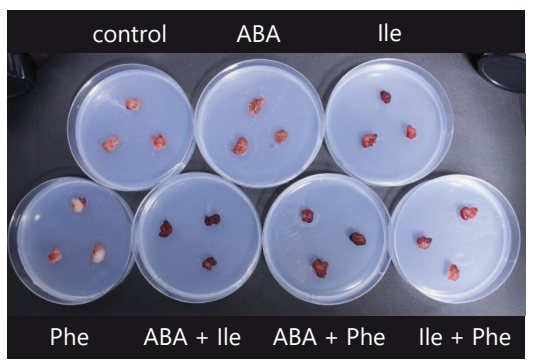

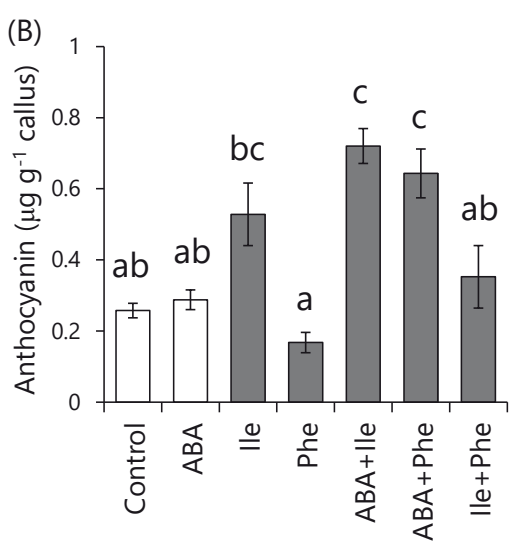

Figure 2. Isoleucine and phenylalanine enhanced low-concentration ABA-mediated anthocyanin accumulation in VR cells. VR cells were treated with $10 \mathrm{mM}$ isoleucine or phenylalanine, and $0.1 \mathrm{mM} \mathrm{ABA}$. (A) VR cells on day 5 post treatment. (B) Anthocyanin contents in VR cells. Bars indicate means \pm standard errors from three independent experiments. Different letters above the columns indicate statistically significant differences $(p<0.05)$. Ile - isoleucine, Phe - phenylalanine
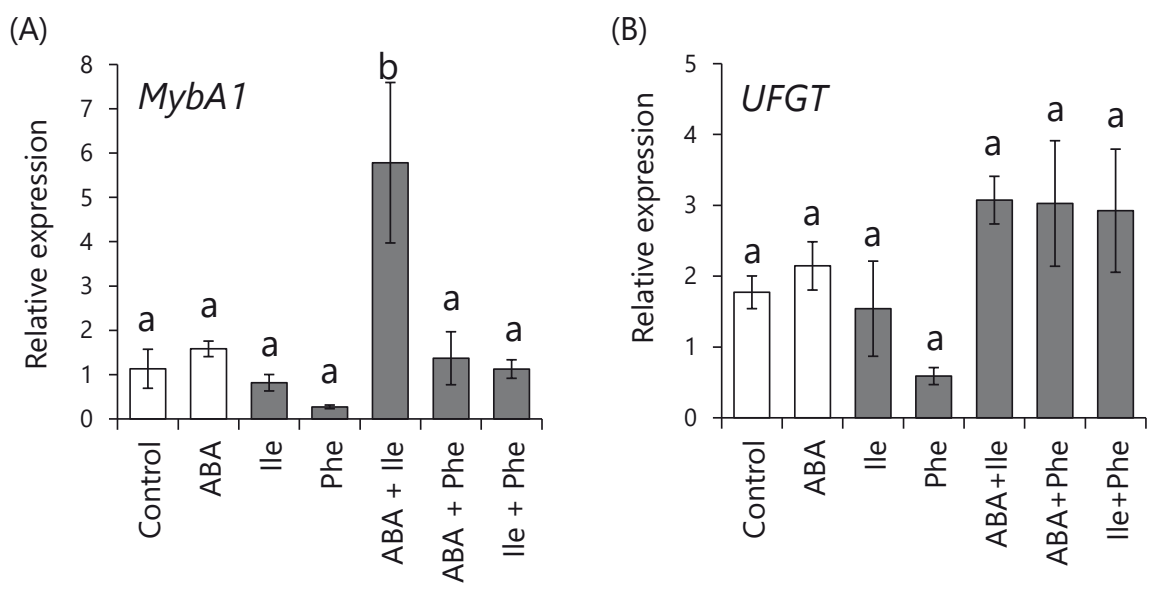

Figure 3. Transcription of MybA1 and UFGT in VR cells treated with $10 \mathrm{mM}$ isoleucine or phenylalanine, and 0.1 mM ABA. The transcription levels of MybA1 (A) and UFGT (B) in VR cells on day 5 post treatment were estimated by real-time RT-PCR. Data were calculated as gene expression relative to actin expression. Bars indicate means \pm standard errors from three independent experiments. Different letters above the columns indicate statistically significant differences $(p<0.05)$. Ile - isoleucine, Phe - phenylalanine

(A)

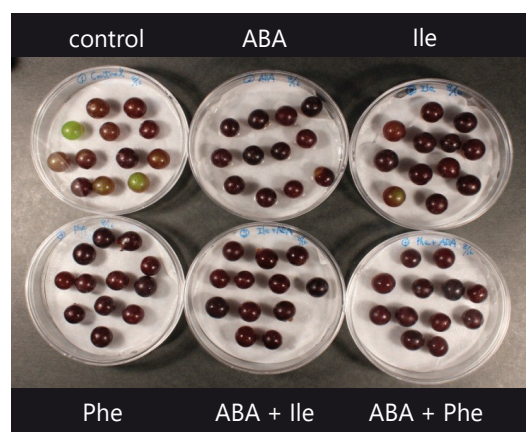

(B)

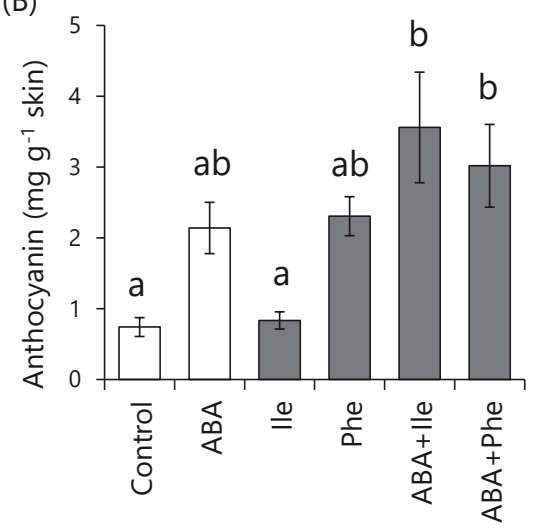

Figure 4. Anthocyanin contents in the skins of detached grape berries treated with isoleucine or phenylalanine, and a low concentration of ABA. The detached grape berries were treated with $10 \mathrm{mM}$ isoleucine or phenylalanine, and $1 \mathrm{mM}$ ABA. (A) Detached grape berries on day 8 post treatment. (B) Anthocyanin contents in the skins of detached grape berries. Bars indicate means \pm standard errors from four independent experiments. Different letters above the columns indicate statistically significant differences $(p<0.05)$. Ile - isoleucine, Phe - phenylalanine 
MybA1 and UFGT transcription in VR cells receiving combination treatment with isoleucine or phenylalanine, and $A B A$

UFGT, which catalyzes the glycosylation of anthocyanidin, and MybA1, which is a transcription factor for UFGT, are crucial determinants of anthocyanin accumulation in grape berry skin (Boss et al., 1996; Kobayashi et al., 2004). To determine whether the combination treatment with isoleucine or phenylalanine, and ABA upregulated MybA1 or UFGT expression, the transcriptional profiles of those genes in VR cells treated with isoleucine or phenylalanine, and ABA were clarified (Fig. 3). ABA at a low concentration $(0.1 \mathrm{mM}) \mathrm{did}$ not upregulate MybA1 expression in VR cells (Fig. 3A). Isoleucine or phenylalanine alone also did not affect MybA1 expression in VR cells. In contrast, VR cells treated with a combination of isoleucine and ABA showed significantly upregulated MybA1 expression compared with the control and ABAtreated VR cells, whereas VR cells treated with a combination of phenylalanine and ABA did not show any upregulation of MybA1 expression. On the other hand, no significant difference in UFGT expression was observed among the variously treated VR cells, although the combination treatment with isoleucine or phenylalanine, and $\mathrm{ABA}$ led to a slight upregulation of UFGT expression (Fig. 3B). Simultaneous treatment with isoleucine and phenylalanine affected neither MybA1 nor UFGT expression in VR cells.

\section{Effect of combination treatment with isoleucine or phenylalanine, and $A B A$ on anthocyanin content in detached grape berry}

Detached grape berries were used to investigate whether the combination treatment with isoleucine or phenylalanine, and ABA enhanced grape berry coloration (Fig. 4). No difference in skin anthocyanin content was observed between the control grape berries and grape berries treated with a low concentration (1 mM) of ABA (Fig. 4B). The combination treatment with isoleucine or

(A)
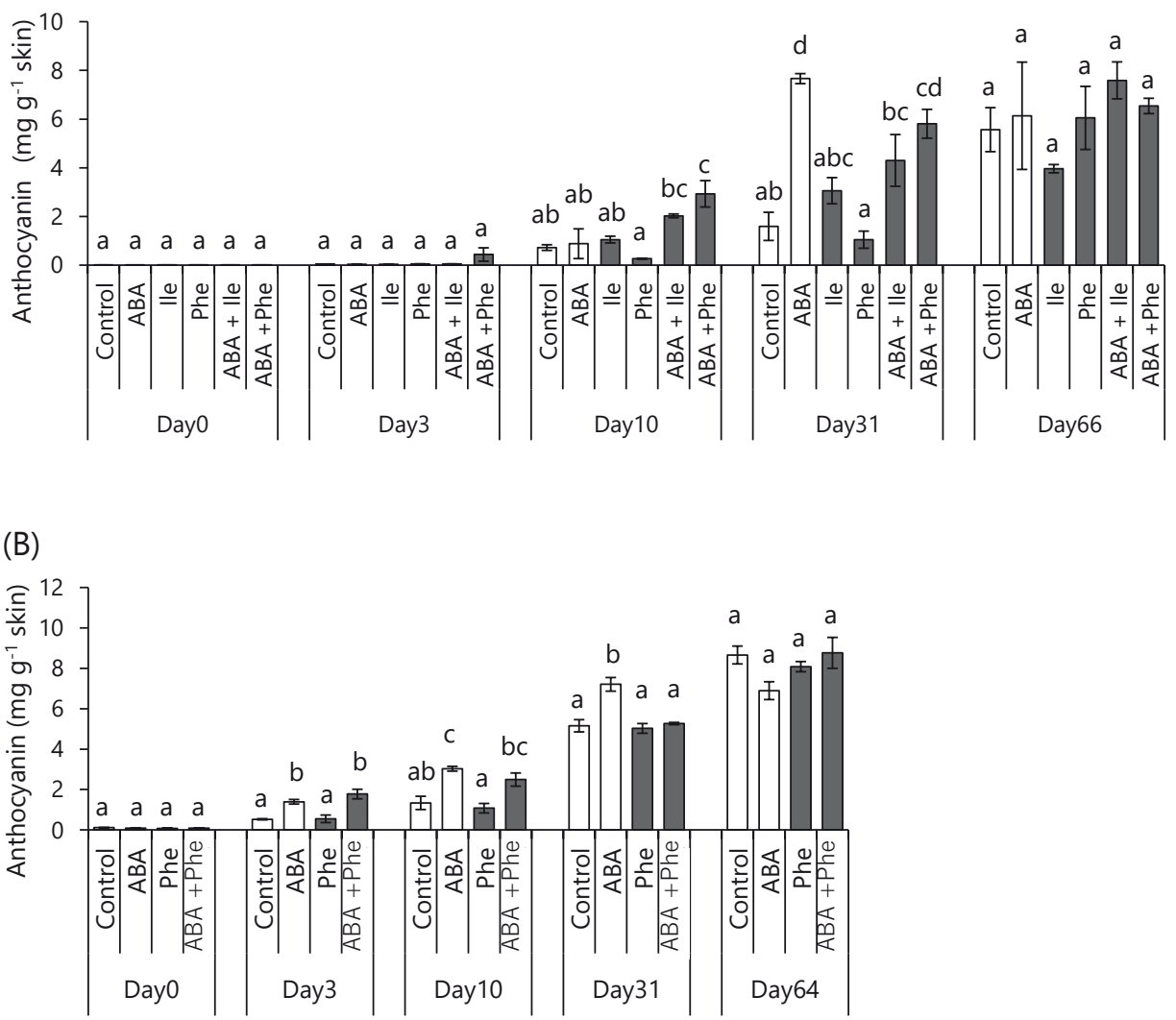

Figure 5. Anthocyanin contents in grape berry skins of field-grown grapevines treated with isoleucine or phenylalanine, and a low concentration of ABA. Grape bunches on field-grown grapevines were dipped into $10 \mathrm{mM}$ isoleucine or phenylalanine, with $380 \mu \mathrm{M}$ ABA. (A) 2015 growing season. (B) 2017 growing season. Anthocyanin contents in berry skins were measured according to Materials and Methods. Bars indicate means \pm standard errors from three bunches. Different letters above the columns indicate statistically significant differences $(p<0.05)$. Ile - isoleucine, Phe phenylalanine, Day 0 - beginning of treatment 
phenylalanine, and ABA significantly increased the anthocyanin content in grape berry skin as compared with control grape berry skin. Taken together, the results again suggested that exogenous isoleucine and phenylalanine, used separately, interacted with a low concentration of ABA-mediated anthocyanin accumulation in grape berry skins.

\section{Effect of combination treatment with isoleucine or phenylalanine, and $A B A$ on anthocyanin content in berry of field-grown grapevine}

Field-grown grapevines were treated with a combination of isoleucine or phenylalanine, and a low concentration of ABA $(380 \mu \mathrm{M})$ in two growing seasons. Sugar and total acid contents were comparable among the treatments in both years (data not shown). In the 2015 growing season, the combination of isoleucine or phenylalanine, and ABA accelerated anthocyanin accumulation in grape berry skin of field-grown grapevine (Fig. 5A and Fig. 6). Enhanced anthocyanin accumulation by the combination of phenylalanine and $\mathrm{ABA}$ was observed on day 10 post application. On day 31 post application, the grape berries treated with the combination of phenylalanine and $\mathrm{ABA}$ accumulated more anthocyanin than those of the control grapevines, although the application of $\mathrm{ABA}$ only also enhanced anthocyanin accumulation. Also, although without a significant difference, there was a certain tendency for the combination

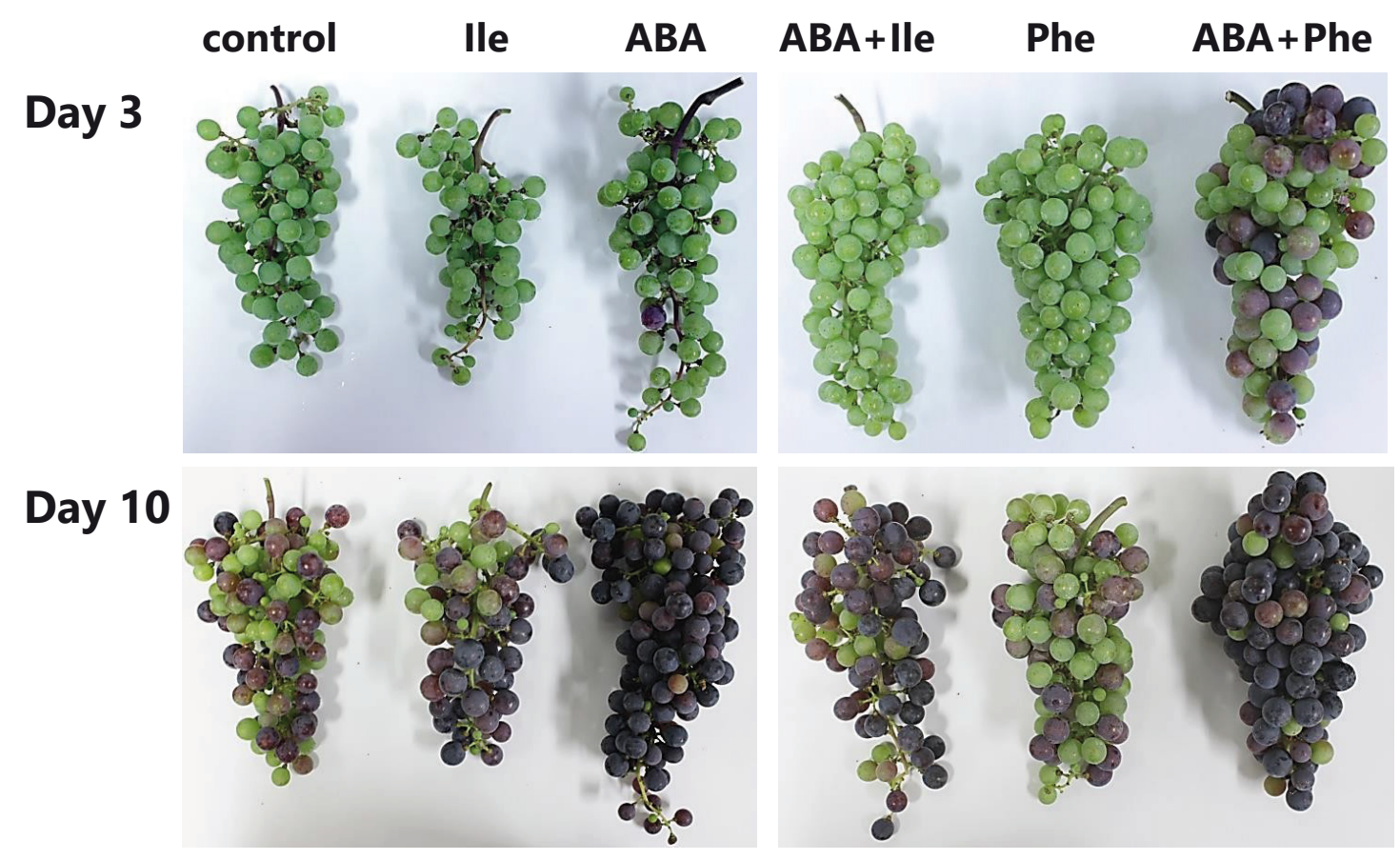

Figure 6. Photographs of grape bunches treated in the 2015 growing season. The photographs show arbitrarily collected bunches on days 3 and 10 post treatment. Ile - isoleucine, Phe - phenylalanine

Day 3

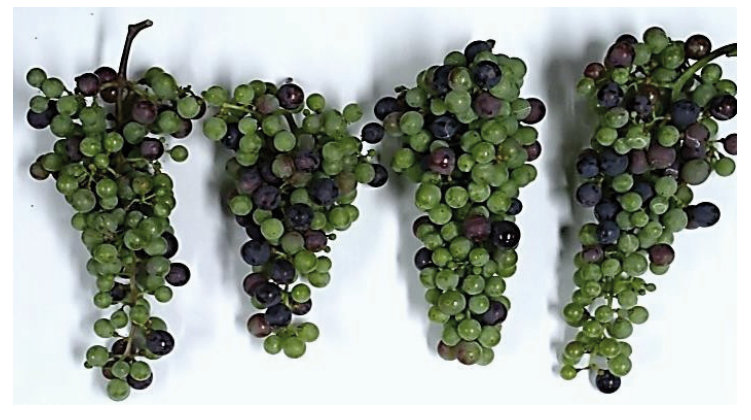

control ABA Phe ABA+Phe
Day 10

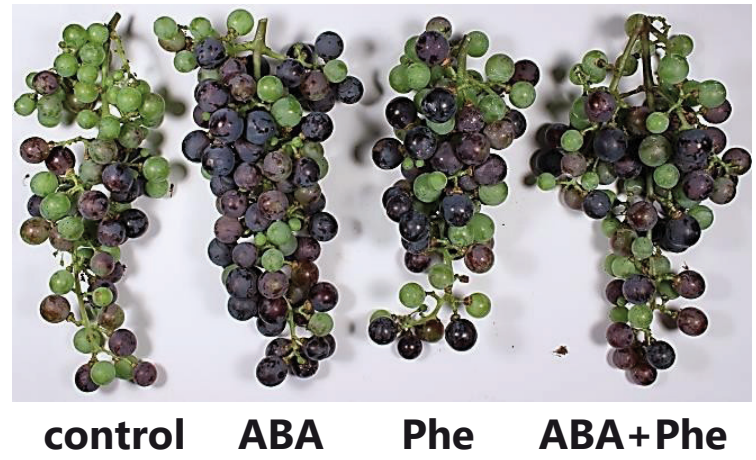

Figure 7. Photographs of grape bunches treated in the 2017 growing season. The photographs show arbitrarily collected bunches on days 3 and 10 post treatment. Phe - phenylalanine 
of isoleucine and ABA to induce accumulation of more anthocyanin than in the control grapevines (Fig. 5A, day 31 post application).

In response to the experimental data from the 2015 growing season, the field experiment in the 2017 growing season aimed at a re-evaluation of the combination effect of phenylalanine and ABA on anthocyanin accumulation. The acceleration of anthocyanin accumulation by the combination treatment with phenylalanine and ABA was reconfirmed in the 2017 growing season (Fig. 5B and Fig. 7). Although a low concentration of ABA stimulated anthocyanin accumulation, the combination of phenylalanine and ABA also accelerated anthocyanin accumulation on days 3 and 10 post application as compared with control grapevines. In both seasons, the effects on anthocyanin accumulation became negligible at harvest (day 66 for 2015 and day 64 for 2017).

Taken together, the results suggested that exogenous isoleucine and phenylalanine, used separately, interacted with ABA-mediated anthocyanin accumulation in the berries of fieldgrown grapevines when ABA activity was inadequate.

\section{DISCUSSION}

ABA regulates plant growth and fruit maturation. In grape berry, endogenous ABA increases around véraison, and then sugar accumulation and berry colour development begin (Kataoka et al., 1982; Peppi et al., 2008; Wheeler et al., 2009). Exogenous ABA application to grapevine stimulates berry ripening and anthocyanin accumulation in grape berry (Koyama et al., 2010). However, because synthetic ABA is expensive, ABA has not been widely applied in viticulture. In the present study, we demonstrated that the combination treatment with isoleucine or phenylalanine, and a low concentration of ABA synergistically increased the anthocyanin content in grape berry. Foliar application of phenylalanine did not affect microbial diversity in grape must and had a negative effect on wine fermentation (González-Arenzana et al., 2017). As ABA application could be reduced by using a combination of inexpensive isoleucine or phenylalanine, and ABA, the combined treatment with isoleucine or phenylalanine, and a low concentration of ABA would be a new and practical tool for improving anthocyanin accumulation in grape berry.

How does isoleucine or phenylalanine improve ABA-mediated anthocyanin accumulation? Phenyl- alanine is the initial compound in anthocyanin biosynthesis via the phenylpropanoid pathway (Dixon et al., 2002). Exogenous phenylalanine increases endogenous phenylalanine (GardeCerdán et al., 2014) and accelerates anthocyanin accumulation (Portu et al., 2015) in grapevine. In the present study, the combination treatment with phenylalanine and a low concentration of $\mathrm{ABA}$ did not upregulate MybA1 or UFGT expression in grape berry. A recent study suggested the presence of mechanisms, such as enzymatic regulation or anthocyanin transport, for determining the anthocyanin accumulation in grape berry skins, since the transcription of genes in the flavonoid biosynthesis pathway was not always temporally correlated with the increase in anthocyanin concentration (Gaiotti et al., 2018). ABA increases phenylalanine ammonia-lyase (PAL) expression (Ban et al., 2003). As PAL catalyzes the conversion of phenylalanine into trans-cinnamic acid in the upstream of the phenylpropanoid pathway (MacDonald and D'Cunha, 2007), the combination treatment with phenylalanine and a low concentration of ABA may promote anthocyanin synthesis by cooperatively stimulating the upstream of the phenylpropanoid pathway but not the anthocyanin biosynthesis pathway.

Conversely, the combination treatment with isoleucine and a low concentration of ABA upregulated MybA1 expression in grape berry, resulting in the promotion of anthocyanin accumulation in grape berry. Although isoleucine might activate the anthocyanin biosynthesis pathway directly or indirectly, so far, we have no evidence to support this hypothesis. Indanoyl isoleucine accelerated anthocyanin accumulation in grape cells (Cai et al., 2012). Indanoyl isoleucine is a synthetic elicitor that plays the role of a mimic signal in plant resistance toward insect attacks and affects the production of plant secondary metabolites (Schuler et al., 2004). Jasmonate isoleucine is a conjugate of jasmonate and isoleucine, and plays an important role in the jasmonate signalling pathway, including colour formation in plants (Rudell et al., 2002; Shan et al., 2009). Thus, exogenous isoleucine may act as an activator of the anthocyanin biosynthesis pathway through the formation of isoleucine conjugates or their derivatives in grapevine. Further studies employing pulse-chase experiments with radiolabelled isoleucine would reveal the dynamics of exogenous isoleucine in grapevines. Global warming affects both the quality and production of grape berry and wine (De Orduña, 2010). A high 
temperature caused low anthocyanin accumulation in grape berry (Mori et al., 2007; Spayd et al., 2002; Tarara et al., 2008). Although various viticultural practices aimed at improving anthocyanin content in grape berry have been developed (Carreño et al., 1998; Koshita et al., 2011; Koyama et al., 2010; Peppi et al., 2008; Tardaguila et al., 2010), those practices are time-consuming and not sufficiently effective. Here, we demonstrated that both isoleucine and phenylalanine enhanced low-concentration ABAmediated anthocyanin accumulation in grape berry. The difference in the experimental data between the 2015 and 2017 growing seasons suggested that environmental and weather conditions affected the effects of the combination treatment with isoleucine or phenylalanine, and a low concentration ABA on anthocyanin accumulation in grape berry. A previous study had reported that phenylalanine did not always improve grape coloration and that the effect of phenylalanine on anthocyanin accumulation might be limited by certain conditions, such as plant nitrogen requirement (Portu et al., 2017). Therefore, further field trials are necessary to identify the best conditions, including the cultivation environment, the doses of isoleucine, phenylalanine, and ABA, and the timing of application, to ensure that the combination treatment with isoleucine or phenylalanine, and a low concentration of ABA works effectively in grapevines. Also, so far, we have been able to demonstrate some positive results that anthocyanin accumulation in grape berry treated by the combination treatment with isoleucine or phenylalanine, and a low concentration ABA contributes to wine colour. Future studies employing wine production using treated berries would reveal the suitability of the combination treatment as a viticultural practice.

\section{CONCLUSIONS}

As global warming has resulted in a loss of anthocyanin content in grape berry skin, viticulturists face the tough challenge of maintaining grape berry skin colour. The goal of the present study was to examine whether the application of amino acids to grapevines would maintain and improve grape berry skin colour. Among the amino acids tested, both isoleucine and phenylalanine enhanced anthocyanin synthesis in grape cell cultures. Also, the combination treatment with isoleucine or phenylalanine, and a low concentration of ABA enhanced anthocyanin accumulation in both grape cell cultures and skins of detached grape berries. Our experiments suggested that exogenous application of either isoleucine or phenylalanine interacted with ABAmediated anthocyanin accumulation in grape berry skins of field-grown grapevines. Practical techniques to maintain and improve grape berry skin colour are required by viticulturists. We propose that the combination treatment of isoleucine or phenylalanine, and a low concentration of ABA could be a new tool for maintaining or improving anthocyanin accumulation in grape berry skins.

\section{AUTHOR CONTRIBUTIONS}

T.H., Y.C. and D.I. - developed the concept and designed the experiment; T.H. Y.C. and S.S. collected data and performed analyses; T.H., D.I. and S.S. - analyzed the data and wrote the paper.

\section{CONFLICT OF INTEREST}

The Authors declare no conflict of interest.

\section{REFERENCES}

Arita K., Honma T., Suzuki S., 2017. Comprehensive and comparative lipidome analysis of Vitis vinifera cv. Pinot Noir and Japanese indigenous $V$. vinifera $\mathrm{cv}$. Koshu grape berries. PLoS ONE 12, e0186952.

Bakker J., Preston N.W., Timberlake C.F., 1986. The determination of anthocyanins in aging red wines: comparison of HPLC and spectral methods. Am. J. Enol. Vitic. 37, 121-126.

Ban T., Ishimaru M., Kobayashi S., Goto-Yamamoto N., Horiuchi S., 2003. Abscisic acid and 2,4-dichlorophenoxyacetic acid affect the expression of anthocyanin biosynthetic pathway genes in 'Kyoho' grape berries. J. Hortic. Sci. Biotechnol. 78, 586-589.

Boss P.K., Davies C, Robinson S.P., 1996. Analysis of the expression of anthocyanin pathway genes in developing Vitis vinifera L. cv Shiraz grape berries and the implications for pathway regulation. Plant Physiol. 111, 1059-1066.

Brar H.S., Singh Z., Swinny E., Cameron I., 2008. Girdling and grapevine leafroll associated viruses affect berry weight, colour development and accumulation of anthocyanins in 'Crimson Seedless' grapes during maturation and ripening. Plant Sci. $175,885-897$.

Cai Z., Knorr D., Smetanska I., 2012. Enhanced anthocyanins and resveratrol production in Vitis vinifera cell suspension culture by indanoylisoleucine, N-linolenoyl-L-glutamine and insect saliva. Enzyme Microb. Technol. 50, 29-34.

Calvo P., Nelson L., Kloepper J.W., 2014. Agricultural uses of plant biostimulants. Plant Soil 383, 3-41.

Carreño J., Faraj S., Martinez A., 1998. Effects of girdling and covering mesh on ripening, colour and 
fruit characteristics of 'Italia' grapes. J. Hortic. Sci. Biotech. 73, 103-106.

Castellarin S.D., Gaspero G.D., Marconi R., Nonis A., Peterlunger E., Paillard S., et Al., 2006. Colour variation in red grapevines (Vitis vinifera L.): genomic organisation, expression of flavonoid 3'-hydroxylase, flavonoid 3',5'-hydroxylase genes and related metabolite profiling of red cyanidin-/blue delphinidin-based anthocyanins in berry skin. BMC Genomics 7, 1471-1488.

Castellarin S.D., Gaspero D.G., 2007. Transcriptional control of anthocyanin biosynthetic genes in extreme phenotypes for berry pigmentation of naturally occurring grapevines. BMC Plant Biol. 30, 46-55.

De Orduña R.M., 2010. Climate change associated effects on grape and wine quality and production. Food Res. Int. 43, 1844-1855.

Deluc L.G., Grimplet J., Wheatley M.D., Tillett R.L., Quilici D.R., Osborne C., ET AL., 2007. Transcriptomic and metabolite analyses of Cabernet Sauvignon grape berry development. BMC Genomics 8, 429.

Dixon R.A., Achnine L., Kota P., Liu C.J., Reddy M.S., WANG L., 2002. The phenylpropanoid pathway and plant defence-a genomics perspective. Mol. Plant Pathol. 3, 371-390.

Enoki S., Hattori T., Ishiai S., Tanaka S., Mikami M., Arita K., eT AL., 2017. Vanillylacetone upregulates expression of genes leading to anthocyanin accumulation by inducing endogenous abscisic acid in grape cell cultures. J. Plant Physiol. 219, 22-27.

Gagné S., Estève K., Deytieux C., Saucier C., Gény L., 2006. Influence of abscisic acid in triggering "véraison" in grape berry skins of Vitis vinifera L. cv. Cabernet Sauvignon. J. Int. Sci. Vigne Vin. 40, 7-14.

Gaiotti F., Pastore C., Filippetti I., Lovat L., Belfiore N., Tomasi D., 2018. Low night temperature at véraison enhances the accumulation of anthocyanins in Corvina grapes (Vitis vinifera L.). Sci. Rep. 8, 8719.

Garde-Cerdán T., López R., Portu J., GonzÁlezArenzana L., López-Alfaro I., Santamaría P., 2014. Study of the effects of proline, phenylalanine, and urea foliar application to Tempranillo vineyards on grape amino acid content. Comparison with commercial nitrogen fertilisers. Food Chem. 163, 136-141.

GĄSTOŁ M., 2015. Vineyard performance and fruit quality of some interspecific grapevine cultivars in cool climate conditions. Folia Hort. 27, 21-31.

González-Arenzana L., Portu J., López R., Garijo P., Garde-Cerdán T., López-Alfaro I., 2017. Phenylalanine and urea foliar application: Effect on grape and must microbiota. Int. J. Food Microbiol. 245, 88-97.

Guidoni S., Allara P., Schubert A., 2002. Effect of cluster thinning on berry skin anthocyanin composition of Vitis vinifera cv. Nebbiolo. Am. J. Enol. Vitic. 53, 224-226.

Jones G.V., White M.A., Cooper O.R., Storchmann K., 2005. Climate change and global wine quality. Clim. Change 73, 319-43.

Kataoka I., Sugiura A., Utsunomiya N., Tomana T., 1982. Effect of abscisic acid and defoliation on anthocyanin accumulation in Kyoho grapes (Vitis vinifera L. $\times$ V. labruscana Bailey). Vitis 21, 325-332.

Kennedy J.A., SAucier C., Glories Y., 2006. Grape and wine phenolics: history and perspective Am. J. Enol. Vitic. 57, 239-248.

Kobayashi S., Goto-Yamamoto N., Hirokawa H., 2004. Retrotransposon-induced mutations in grape skin color. Science 304, 982.

Koshita Y., Yamane T., Yakushiji H., Azuma A., Mitani N., 2011. Regulation of skin color in 'Aki Queen' grapes: interactive effects of temperature, girdling, and leaf shading treatments on coloration and total soluble solids. Sci. Hortic. 129, 98-101.

Koyama K., Sadamatsu K., Goto-Yamamoto N., 2010. Abscisic acid stimulated ripening and gene expression in berry skins of the Cabernet Sauvignon grape. Funct. Integr. Genomics 10, 367-381.

MacDonald M.J., D’Cunha G.B., 2007. A modern view of phenylalanine ammonia lyase. Biochem. Cell Biol. 85, 273-282.

Matus J.T., Loyola R., Vega A., Neira A.P., Bordeu E., Johnson P.A., ET AL., 2009. Post-veraison sunlight exposure induces MYB-mediated transcriptional regulation of anthocyanin and flavonol synthesis in berry skins of Vitis vinifera. J. Exp. Bot. 60, 853-867.

Mikami M., Mori D., Masumura Y., Aoki Y., Suzuki S., 2017. Electrical stimulation: an abiotic stress generator for enhancing anthocyanin and resveratrol accumulation in grape berry. Sci. Hortic. 226, $285-$ 292.

Mori K., Goto-Yamamoto N., Kitayama M., Hashizume K., 2007. Loss of anthocyanins in red-wine grape under temperature. J. Exp. Bot. 58, 1935-1945.

Mori K., Sugaya S., Gemma H., 2005. Decreased anthocyanin biosynthesis in grape berries grown under elevated night temperature condition. Sci. Hortic. 105, 319-330.

PepPi M.C., Walker M.A., Fidelibus M.W., 2008. Application of abscisic acid rapidly upregulated UFGT gene expression and improved color of grape berries. Vitis 47, 11-24.

Pilati S., Bagagli G., Sonego P., Moretto M., Brazzale D., Castorina G., et al., 2017. Abscisic acid is a major regulator of grape berry ripening onset: new insights into ABA signaling network. Front. Plant Sci. 8, 1093.

Portu J., Gonzalez-Arenzana L., HermosínGutiérrez I., Santamaría P., Garde-Cerdan T., 2015. Phenylalanine and urea foliar applications to grapevine: Effect on wine phenolic content. Food Chem. 180, 55-63. 
Portu J., López R., Baroja E., SANTAMaría P., GARdeCerdán, T., 2016. Improvement of grape and wine phenolic content by foliar application to grapevine of three different elicitors: Methyl jasmonate, chitosan, and yeast extract. Food Chem. 201, 213-221.

Portu J., Santamaría P., LóPez R., Garde-Cerdán T., 2017. Phenolic composition of Tempranillo grapes following foliar applications of phenylalanine and urea: A two-year study. Sci. Hortic. 219, 191-199.

Romero-Cascales I., Ortega-Regules A., López-Roca J.M., Fernández-Fernández J.I., Gómez-Plaza E., 2005. Differences in anthocyanin extractability from grapes to wines according to variety. Am. J. Enol. Vitic. 56, 212-219.

Rudell D.R., Mattheis J.P., Fan X., Fellman J.K., 2002. Methyl jasmonate enhances anthocyanin accumulation and modifies production of phenolics and pigments in Fuji' Apples. J. Am. Soc. Hortic. Sci. 127, 435-441.

Schuler G., Mithofer A., Baldwin I.T., Berger S., Ebel J., Santos J.G., et AL., 2004. Coronalon: A powerful tool in plant stress physiology. FEBS Lett. 563, 17-22.

Shan X., Zhang Y., Peng W., Wang Z., XIE D., 2009. Molecular mechanism for jasmonate-induction of anthocyanin accumulation in Arabidopsis. J. Exp. Bot. 60, 3849-3860.

Smit I., Pfliehinger M., Binner A., Grossmann M., Horst W.J., LöHnertz O., 2014. Nitrogen fertilisation increases biogenic amines and amino acid concentrations in Vitis vinifera var. Riesling musts and wines. J. Sci. Food Agric. 94, 2064-2072.
Spayd S.E., Tarara J.M., Mee D.L., Ferguson J.C., 2002. Separation of sunlight and temperature effects on the composition of Vitis vinifera cv. Merlot berries. Am. J. Enol. Vitic. 53, 171-182.

Tarara J.M., Lee J., Spayd S.E., Scagel C.F., 2008. Berry temperature and solar radiation alter acylation, proportion, and concentration of anthocyanin in Merlot grapes. Am. J. Enol. Vitic. 59, 235-247.

Tardaguila, J., de Toda, F.M., Poni, S., Diago, M.P., 2010. Impact of early leaf removal on yield and fruit and wine composition of Vitis vinifera L. Graciano and Carignan. Am. J. Enol. Vitic. 61, 372-381.

Wheeler S., Loveys B., Ford C., Davies C., 2009. The relationship between the expression of abscisic acid biosynthesis genes, accumulation of abscisic acid and the promotion of Vitis vinifera L. berry ripening by abscisic acid. Aust. J. Grape Wine Res. 15, 195204.

Yamakawa T., Kato S., Ishida K., Kodama T., Minoda Y., 1983. Production of anthocyanins by Vitis cells in suspension culture. Agric. Biol. Chem. 47, 2185 2191.

Yokotsuka K., Nagao A., Nakazawa K., Sato M., 1999. Changes in anthocyanins in berry skins of Merlot and Cabernet Sauvignon grapes grown in two soils modified with limestone or oyster shell versus a native soil over two years. Am. J. Enol. Vitic. 50, $1-12$.

Received September 7, 2018; accepted December 11, 2018 